\title{
Storing the Electric Energy Produced by an AC Generator
}

P. Simeão Carvalho, Departamento de Física e Astronomia, Faculdade de Ciências da Universidade do Porto and Instituto de Física dos Materiais da Universidade do Porto, Portugal

Ana Paula Lima, Escola Secundária Dr. Manuel Gomes de Almeida, Espinho, Portugal

Pedro Simeão Carvalho, Departamento de Engenharia Informática, Faculdade de Engenharia da Universidade do Porto, Portugal

$\mathrm{P}$ roducing energy from renewable energy sources is nowadays a priority in our society. In many cases this energy comes as electric energy, and when we think about electric energy generators, one major issue is how we can store that energy. In this paper we discuss how this can be done and give some ideas for applications that can serve as a motivation for projects with students.

First consider a dc electric motor, which is capable of producing pulsating $\mathrm{dc}$ when operated as a generator. When inserted in an electric circuit such as the one shown in Fig. 1, the LED (light emitting diode) indicates that electric current is circulating between points $\mathrm{P}$ and $\mathrm{N}$.

Each time the rotation of the motor is reversed, the electric current circulates in the opposite sense. If we make it happen alternately, this causes the LED in Fig. 1 to blink.

\section{How can we store the electric energy generated by the motor?}

We can store the electric energy produced by the motor, replacing the LED in Fig. 1 with a capacitor (or a battery, according to the use we will give to it): when the capacitor is charged, an electric voltage can be measured in the capacitor by using a voltmeter.

However, there is a major problem with this way of storage when we use an ac generator or when the rotation of the motor ( $\mathrm{dc}$ generator) is reversed: the electric current is also inverted and, as a consequence, the capacitor discharges!

To avoid discharging the capacitor, therefore keeping all the electric energy generated, we need to force the electric current to circulate in the branch of the LED/capacitor always in the same direction. This can be done with a diode bridge (also called a rectification circuit), as shown in Fig. 2. No matter the signal of the emf produced at the output of the generator, we always obtain direct current in the P-N branch (the one with the LED); if instead of an LED we put a capacitor, the electric energy can be stored in it.

In order to show this effect in a very nice application, we have built a prototype consisting of a giant wheel ${ }^{1}$ mounted on a wood platform that could be put floating on a water course. The wheel, with rectangular panels in the inner part serving as shovels to increase the impact of water, was free to rotate in any sense (Fig. 3).

We have then incorporated two rectification circuits, each one with an electric motor that was linked to the periphery of the giant wheel by using a self-adhesive foam:

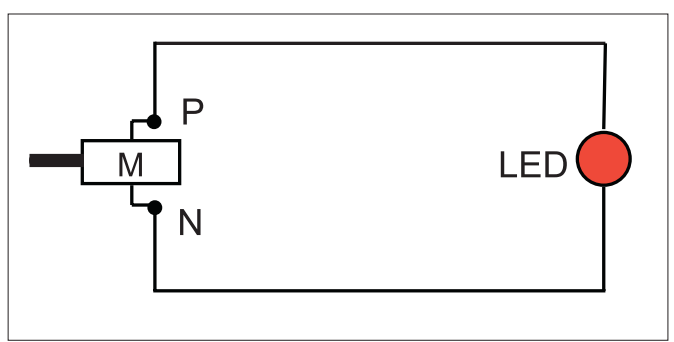

Fig. 1. Scheme of a simple electric circuit with a motor (M) and an LED. Points $\mathrm{P}$ and $\mathrm{N}$ represent the two electric motor contacts.

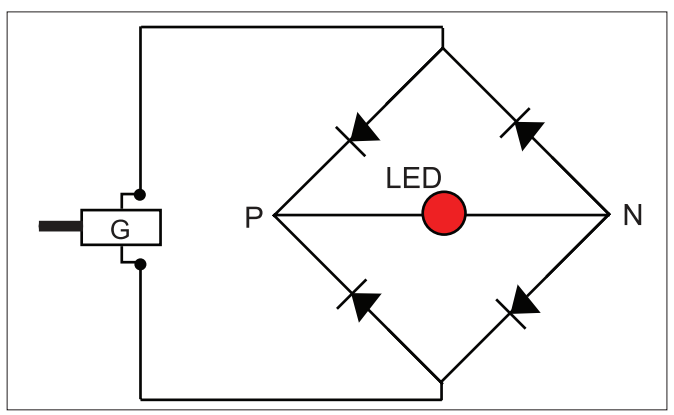

Fig. 2. Schematic representation of a diode bridge connected to a generator (G). Direct current is obtained in the P-N branch.

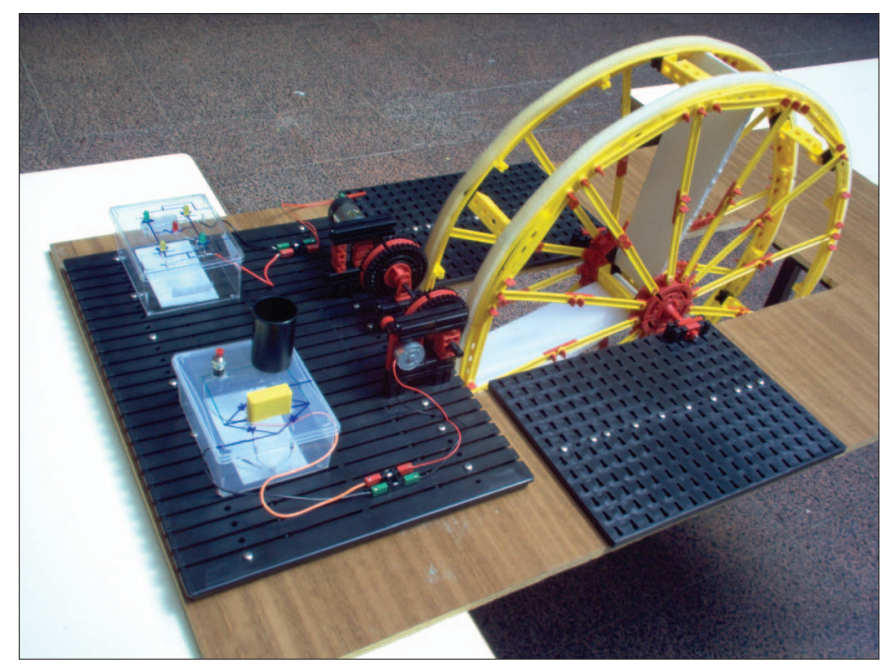

Fig. 3. Giant wheel attached to a wood platform, free to rotate in either direction. Two small electric motors can be seen on the left side, connected to the periphery of the wheel by self-adhesive foam. 

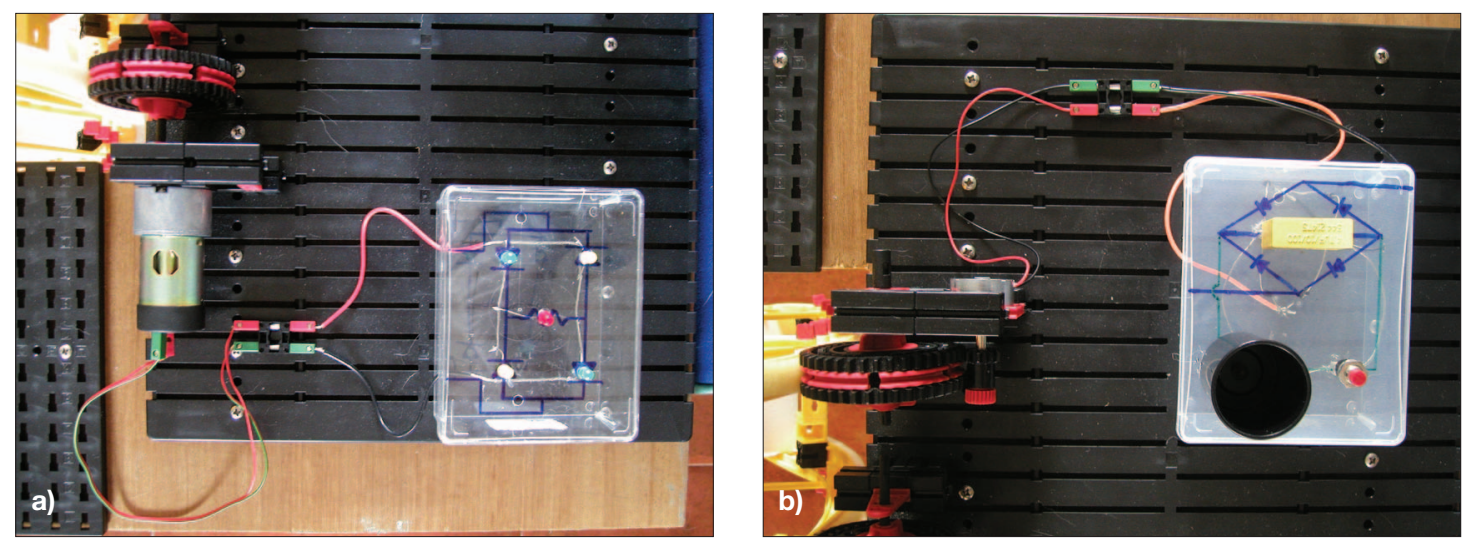

Fig. 4. Photographs of electric circuits including diode bridges: (a) with different colored LED to illustrate the sense of the electric current, and (b) with diodes and a capacitor in the dc branch for electric energy storage. The discharging circuit is also seen, consisting of a red pressure button and an LED inside a small black can.

- Circuit 1: A colored LED circuit to illustrate that dc current was induced in the red LED branch whenever the giant wheel was rotated [Fig. 4(a)]. In this circuit, LED green and yellow blink according to the direction of rotation.

- Circuit 2: A storage circuit where electric energy was accumulated in a capacitor that could later be discharged, by means of a discharging parallel circuit with an LED inside a small black can [Fig. 4(b)].

Regardless of which way the generators turn, the red LED is always lighted (circuit 1) and energy is stored in the capacitor of circuit 2. Used in a bigger scale, this method for capturing energy can be very efficient at taking advantage of renewal sources.

Many situations can therefore be seen as potential energy sources, such as the river's unidirectional flow or the bidirectional flow of water waves at the seashores. ${ }^{2}$ Tides can also be seen as potential energy sources, because when the tide changes the direction of the water flow at the mouth of rivers and bays is reversed, so a bidirectional system such as the one described above could be working uninterruptedly. These are novel ideas for applications to produce continuously electric energy 24 hours per day at (nearly) zero cost.

We are convinced that this can be economically very attractive in countries with a large coastline like Portugal, taking advantage of Portuguese rivers and pleasant beaches, one more idea for a better use of natural resources. ${ }^{3}$

\section{References}

1. www.fischertechnik.de.

2. An interesting animation can be seen at the site: www.energiasrenovaveis.com/images/upload/flash/anima_ como_funciona/ondas13.swf.

3. This project was awarded with the third prize in Ideas for Science Fairs, First International Conference Hands on Science, Braga, Portugal, 2006.

Paulo Simeão Carvalho is an assistant professor in the Porto University Physics and Astronomy Department. His research interests are mainly liquid crystals, liquid crystal-polymer composites, and physics education. He has been working in teacher training (initial and continuous) for the last 15 years.

psimeao@fc.up.pt

Ana Paula Lima is a graduate of Porto University and a physics and chemistry teacher. She got her master's degree in physics at Porto University in 2000. She has been a permanent teacher at Escola Secundária Dr. Manuel Gomes de Almeida, Espinho, since 2002. aplima@netcabo.pt

Pedro Simeão Carvalho finished his secondary studies at Escola Secundária Dr. Manuel Gomes de Almeida, Espinho. In 2006 he was awarded the third prize in Ideas for Science Fairs at the International Conference HSCl2006, Braga. He is currently a second-year student in the Master's program in Informatics and Computing Engineering at Porto University.

pesimeao@gmail.com 